\title{
Caracterización del estado antropométrico y de las capacidades físicas de los bailarines del grupo de danza de la Universidad del Atlántico
}

\section{Characterization of the anthropometric state and of the physical capacities of the dancers of the dance group of the Universidad del Atlántico}

Guiliany Andrea Llinás-Malvido ${ }^{1}$, Amalia Villamizar-Navarro ${ }^{1}$, Samuel Villamarín-Menzaํㅛ

1Universidad del Atlántico. Barranquilla, Colombia; guily0616@gmail.com; amaliavillamizar@mail. uniatlantico.edu.co; samuelvillamarin@mail.uniatlantico.edu.co

Cómo citar: Llinás-Malvido, G.A.; Villamizar-Navarro, A.; Villamarín-Menza, S. 2021. Caracterización del estado antropométrico y de las capacidades físicas de los bailarines del grupo de danza de la Universidad del Atlántico. Rev. Digit. Act. Fis. Deport. 7(1):e1501. http://doi. org/10.31910/rdafd.v7.n1.2021.1501

Artículo de acceso abierto publicado por Revista Digital: Actividad Física y Deporte, bajo una licencia Creative Commons CC BY-NC 4.0

Publicación oficial de la Universidad de Ciencias Aplicadas y Ambientales U.D.C.A, Institución de Educación Superior Acreditada de Alta Calidad por el Ministerio de Educación Nacional.

Recibido: 17 de marzo de 2020 Aceptado: 28 de octubre de 2020 Editado por: Álvaro José Gracia Díaz

\section{RESUMEN}

Introducción: La investigación busca explorar y describir las capacidades físicas y antropométricas básicas que presentan los bailarines del grupo de danza de la Universidad del Atlántico. Objetivo: Caracterizar el estado antropométrico y las capacidades físicas de un grupo de bailarines pertenecientes al equipo de danza de la Universidad del Atlántico, en Barranquilla. Metodología: El proceso investigativo implementó un enfoque de investigación cuantitativa, de tipo exploratorio, descriptivo y transversal. Resultados: En la variable del IMC, se encontraron valores normales, todos tenían entre 20,20 y 24,22 , en este indicador. Se halló un \% grasa por 3 pliegues, de $24,91 \%$, para las mujeres y para los hombres, de $9 \%$; además, un $\%$ de grasa por 7 pliegues para mujeres, de $25,50 \%$ y para hombres, de $8 \%$. En los resultados promedio del test de fuerza, el flexo extensión de codos para mujeres, 20 y para hombres, 28 repeticiones; abdominales para mujeres, 19 repeticiones y para hombres, 23; salto alto para mujeres $0,42 \mathrm{~m}$ y para hombres, $0,49 \mathrm{~m}$. En el test de Cooper, los resultados fueron de $30 \mathrm{ml} / \mathrm{kg} / \mathrm{min}$, para mujeres y para hombres, de $39 \mathrm{ml} / \mathrm{kg} / \mathrm{min}$. Finalmente, en el test de flexibilidad los datos promedios de las mujeres fueron de $+14 \mathrm{~cm}$, mientras que para los hombres fueron de $+13 \mathrm{~cm}$. Conclusión: La preparación de los bailarines del grupo de danzas debe incluir la evaluación frecuente de las características antropométricas y motoras.

Palabras clave: Antropometría; Capacidad física; Danza; Flexibilidad; Resistencia aeróbica.

\section{ABSTRACT}

Introduction: The research seeks to explore and describe the basic physical and anthropometric capacities presented by dancers from the dance group of the Universidad del Atlántico. Objective: To characterize the anthropometric state and physical 
capacities of a group of dancers belonging to the dance team of the Universidad del Atlántico, in Barranquilla. Methodology: The research process implemented a quantitative, exploratory, descriptive, and cross-sectional research approach. Results: In the BMI variable, normal values were found, all of which were between 20.20 and 24.22 in this indicator. A fat $\%$ for 3 folds was found: $24.91 \%$ for women and for men of $9 \%$, in addition, a\% fat for 7 folds for women of $25.50 \%$ and for men of $8 \%$. In the average results of the strength test, the flexion elbow extension for women 20 and for men 28 repetitions; abs for women 19 repetitions and for men 23; high jump for women $0.42 \mathrm{~m}$ and for men $0.49 \mathrm{~m}$. In the Cooper test the results were $30 \mathrm{ml} / \mathrm{kg} /$ min for women and for men: $39 \mathrm{ml} / \mathrm{kg} / \mathrm{min}$. Finally, in the flexibility test, the average data for women was $+14 \mathrm{~cm}$, while for men it was $+13 \mathrm{~cm}$. Conclusion: The preparation of dancers in the dance group should include frequent evaluation of anthropometric and motor characteristics.

Keywords: Anthropometry; Physical capacity; Dance, flexibility; Aerobic endurance.

\section{INTRODUCCIÓN}

Desde tiempos inmemoriales, la danza ha sido considerada como una de las actividades de expresión corporal más enriquecedoras para niños, jóvenes y adultos. Debido a su creatividad y capacidad de comunicar, se ha constituido como un medio dinámico de manifestación de sentimientos del ser humano (como el dolor o el amor, expresiones internas-externas, acontecimientos físico-mentales, entre otros), que se fortalece a través de la capacidad y el ingenio de los danzantes. Según mencionan Gardoqui \& Sierra (1994), dicha expresión corporal, se encarga del tratamiento específico y diferenciado de todos los aspectos del movimiento en función de los objetivos de expresión, comunicación y creatividad.

En este mismo sentido, Caballero (2015) afirma que la danza, en todas sus modalidades, supone una actividad de considerable demanda física; de allí que, sin lugar a dudas, sea posible afirmar que para los bailarines su instrumento de expresión artística sea todo su cuerpo (Sánchez, 2016), tal como lo sustentan Irvine et al. (2011).

"La danza precisa de todos los aspectos de la condición física. Una buena condición física es clave a la hora de reducir el riesgo de lesiones, mejorar la interpretación y garantizar una larga carrera como bailarín. Un bailarín sano es aquel con un estado de "bienestar" corporal y mental. Un bailarín con una buena forma física es aquel que puede satisfacer las exigencias de una tarea determinada a un nivel adecuado y pertinente" (Irvine et al. 2011).

Dicha condición física supone la inclusión de una serie de componentes determinados, tales como:

- Capacidad aeróbica: relacionada con niveles de actividad moderada de larga duración.

- Capacidad anaeróbica: relacionada con periodos de actividad máxima cortos, de alta intensidad.

- Resistencia muscular: la capacidad de un músculo de producir un movimiento continuo.

- Fuerza física: la capacidad de un músculo de producir una fuerza máxima una vez.

- Potencia: el aspecto explosivo (relacionado con la velocidad) de la fuerza física.

- Flexibilidad: el rango de movimiento de una articulación junto con la capacidad de estiramiento de un músculo.

- Coordinación neuromuscular: relacionada con el equilibrio, la agilidad, la coordinación y la habilidad.

- Composición del cuerpo: la distribución del peso corporal como una expresión del porcentaje de músculos y grasa.

- Descanso: un periodo de inactividad, para permitir la recuperación y regeneración (Irvine et al. 2011).

Dejando de lado, aquellos postulados que se enfocaban, de manera exclusiva, en la flexibilidad o el peso, elementos regularmente asociados con el sexo, la edad, el nivel técnico artístico alcanzado, el grado de entrenamiento o la ingesta alimentaria (Malina \& Bouchard, 1991).

Con el paso del tiempo, la danza construyó nuevas formas de expresión y lenguaje. Fue así, como se aumentó la versatilidad, la plasticidad y el movimiento 
de los artistas, desarrollando y enriqueciendo las principales condiciones físico-mentales de los danzantes, tales como la estética corporal, el sentido del ritmo, la coordinación de las fuerzas, la ligereza, la resistencia y el equilibrio (Bougart, 1966).

Con ello, se estableció que es indispensable preparar fisiológicamente al deportista, con el ánimo de que pueda cumplir las exigencias del trabajo coreográfico actual, debido a que, su condición física, podría ser el factor que límite o potencie su desarrollo. De esta forma, se determinó que los bailarines, constantemente, se encuentran expuestos a una gran variedad de factores de riesgo (Bronner et al. 2003) y "si se ignora el entrenamiento fisiológico, se podría llegar a obstaculizar el desarrollo de la manifestación artística" (Irvine et al. 2011).

Ahora bien, aunque el acondicionamiento físico puede ser adoptado como un hábito personal es necesario contar con una orientación profesional, que precise la intensidad y la duración de los ejercicios, garantizando la adquisición y la ejecución de cada elemento técnico, además de la resistencia, necesaria durante las arduas jornadas de ensayos, clases y demás situaciones, a las que se enfrentan los danzantes (Marchena et al. 2008).

Por lo anterior, "es muy importante que el entrenamiento esté siempre acorde a la modalidad de danza, paralelo a sus exigencias técnicas, ni subordinado ni tampoco en disonancia con ella" (Vargas, 2009), es decir, que se propenda por un programa eficaz, en el que se incluyan diversos ejercicios que se puedan ejecutar de forma colectiva e individual, siempre y cuando estén adecuados al proceso personal del artista.

Es así, como surgen una serie de investigaciones interesadas en determinar la condición física de los bailarines, mediante la aplicación de pruebas de laboratorio y regímenes de entrenamiento, además de la recolección de datos fisiológicos y psicológicos, acudiendo, también, al conocimiento de las capacidades antropométricas, a través de la implementación de estudios ergonómicos, que permitan evaluar el estado y el desempeño de los mismos.

En este sentido, en los postulados de Arellano \& Yáñez (2009), se afirma que la antropometría es el instrumento idóneo para la determinación y la aplicación de las medidas de cuerpo humano, tanto en reposo como en movimiento, además, de la medición del tejido adiposo (grasa) del mismo. Este campo abarca una variedad de dimensiones, tales como el peso, la estatura (altura de pie), pliegues cutáneos, circunferencias (cabeza, la cintura, entre otros), longitud de las extremidades y anchos (hombro, muñeca, entre otros) (Pate et al. 2012; Vicente, 2015; Mazza, 2000); sin embargo, es necesario implementar también diferentes modelos que, de igual forma, permitan determinar la composición corporal. Uno de los más utilizados, en la actualidad, se basa en la estimación de adiposidad o masa grasa (Ross \& Kerr, 1991; Betancourt \& Díaz, 2006) y en el estudio de la masa libre de grasa (Robergs \& Roberts, 1999; Yasumura et al. 2000).

Por lo anterior, la presente investigación, se enfoca en caracterizar el estado antropométrico y las capacidades físicas motoras de los integrantes del grupo de Danza de la Universidad del Atlántico, mediante la determinación del índice de masa corporal (IMC) y el porcentaje de grasa de los danzantes. De igual manera, se ejecutan valoraciones de fuerza, velocidad gestual, resistencia aeróbica y flexibilidad, con el ánimo de establecer claramente las condiciones en las que se encuentran los artistas.

\section{MATERIALES Y MÉTODOS}

Esta investigación es de tipo exploratorio, descriptivo, transversal y cuantitativo, según Hernández et al. (2007). El presente estudio, se realizó con 7 estudiantes, 3 mujeres, entre los 18 y 23 años y 4 hombres, entre los 24 y 29 años, pertenecientes al Grupo de Danza de la Universidad del Atlántico.

Los bailarines fueron evaluados en los siguientes test físicos:

- Test de Fuerza: Según Lorenzo (2015) es recomendable que la batería de pruebas incluya un mínimo de mediciones de la fuerza de los músculos abdominales, de las extremidades inferiores y superiores.

- Flexo Extensión de Codos: El objetivo del test de fuerza para brazos es medir la capacidad de contracción isométrica de la musculatura flexora de brazos. 
- Abdominales: Con el ánimo de medir la capacidad de contracción de la musculatura abdominal (abdominales rectos y oblicuos).

- Salto Alto: Con el fin, de medir la capacidad de salto vertical.

- Test de Velocidad Gestual: Según Grosser (1992) es la capacidad para efectuar un movimiento o una habilidad motriz en el menor tiempo posible de forma coordinada; se midió la frecuencia de movimiento en la prueba de skipping.

- Test de Cooper: El test de Cooper es una prueba de resistencia aeróbica, que se basa en recorrer la mayor distancia posible en 12 minutos, a una velocidad constante.

- Test de Wells: Según Wells \& Dillon (1952), esta prueba se realiza para medir la flexibilidad, mide rango de movimiento de las articulaciones coxofemoral y de la columna lumbar, así como la capacidad de elongación de musculatura isquiotibial, glútea y extensora de la columna vertebral.

- Índice de masa corporal (IMC). Según George et al. (2007), el IMC es una simple relación entre el peso y la estatura. La teoría que hay de tras de este método es que las proporciones peso/estatura en la población general tiene una relación positiva con él, con el porcentaje de grasa corporal. El IMC, se usa, comúnmente, como indicador de la obesidad y esta correlacionado con un mayor riesgo de enfermedades cardiovasculares. El IMC, se calcula con base en la siguiente fórmula:

$$
\text { IMC = Peso Corporal (kg) / Talla }{ }^{2}(\mathrm{~m})
$$

De igualforma, se midieronvariables antropométricas, como los perímetros (cintura, abdomen y cadera) y pliegues cutáneos (Pectoral, Tricipital, Bicipital, Subscapular, Medio axilar, Abdominal, Suprailíaco y Muslo anterior). Se calcularon los siguientes índices, mediante las siguientes ecuaciones (George et al. 2007): Relación Cintura - Cadera: PCi / PCa (en cm) Índice de estatura al cuadrado:

Hombres: $\mathrm{MCM}(\mathrm{kg})=0,204 \times \mathrm{Ec}^{2}$ (estatura en decímetros) Mujeres: $\mathrm{MCM}(\mathrm{kg})=0,18 \times \mathrm{Ec}^{2}$ (estatura en decímetros)
Porcentaje de grasa: (Peso corporal (kg)-MCM (kg)

$$
\text { (Peso corporal) } \times 100
$$

El porcentaje de grasa, se calculó mediante las ecuaciones descritas por Pollock et al. (1990), en las cuales, se usan 3 o 7 pliegues cutáneos.

Hombres:

$\operatorname{Dc}(\Sigma 3)=1.10938-(0.0008267 \times \Sigma 3)+(0.0000016 x$ $\left.\Sigma 3^{2}\right)-(0.0002574 \times$ edad $)$

$\operatorname{Dc}(\Sigma 7)=1.1120-(0.00043499 \times \Sigma 7)+(0.00000055$ $\left.x \sum 7^{2}\right)-(0.00028826 \times$ edad $)$

Mujeres:

$\operatorname{Dc}\left(\sum 3\right)=1.0994921-\left(0.0009929 \times \sum 3\right)+(0.0000023$ $\left.x \sum 3^{2}\right)-(0.0001392 \times$ edad $)$

$\operatorname{Dc}(\Sigma 7)=1.0970-\left(0.00046971 \times \sum 7\right)+(0.00000056$ $\left.x \sum 7^{2}\right)-(0.00012828 \times$ edad $)$

\section{Dc: Densidad corporal}

$\sum 3$ hombres = Suma de los pliegues cutáneos del pecho, abdomen y muslo ( $\mathrm{mm}$ ).

$\sum 3$ mujeres = Suma de los pliegues cutáneos de tríceps, Suprailíaco y muslo.

$\Sigma 7$ para hombres y mujeres = Suma de los pliegues cutáneos del pecho, medio axilar, tríceps, subescapular, abdomen, Suprailíaco y muslo.

El porcentaje degrasa, se calcula por la ecuación de Siri (1961):

$\% \mathrm{GC}=((4.95 / \mathrm{Dc})-4.50) \times 100)$

Los datos de investigación fueron procesados y analizados utilizando los programas Word y Excel 2010.

\section{RESULTADOS}

Resultados del índice de masa corporal (IMC). En lo que respecta a las tres mujeres bailarinas del grupo de danza de la Universidad del Atlántico, se encontró que la edad de las participantes oscila entre 18 y 23 años, es decir, son adultos jóvenes, con un promedio de 20 años. El peso corporal oscila entre 60 y $64 \mathrm{~kg}$, con un promedio para las mujeres de $62 \mathrm{~kg}$. La talla, se encuentra entre 1,60 y 1,66 metros, con un promedio de 1,62 metros. Según los datos anteriores, se halla un Índice de Masa Corporal (IMC) entre 23,15 y 24,22, 
con un promedio de 23,53, una desviación estándar de 0,60 y un coeficiente de variación bajo de $3 \%$. Teniendo en cuenta, la clasificación de la OMS, las bailarinas investigadas se hallan en un peso normal.

Por su parte, los cuatro hombres bailarines oscilan entre 24 y 29 años, es decir, son adultos con un promedio de 26 años. El peso corporal oscila entre 57 y $73,7 \mathrm{~kg}$, con un promedio para los hombres de $67 \mathrm{~kg}$. La talla, se encuentra entre 1,68 y 1,65 metros, con un promedio de 1,67 metros. Según los datos anteriores, se halla un Índice de Masa Corporal (IMC) de 20,20 y 24,07 , con un promedio de 22 , una desviación estándar de 2 y un coeficiente de variación bajo de $9 \%$. Por lo tanto, los sujetos investigados, también, se encuentran en un peso normal (Tablas 1 y 2 ).

Tabla 1. Variables básicas antropométricas de las bailarinas mujeres del grupo de danza de la Universidad del Atlántico.

\begin{tabular}{|c|c|c|c|c|}
\hline \multicolumn{5}{|c|}{ SEXO FEMENINO } \\
\hline $\mathbf{N}^{\circ}$ & SUJETOS & EDAD & PESO (kg) & TALLA (m) \\
\hline 1 & Sujeto A & 18 & 60 & 1,61 \\
\hline 2 & Sujeto B & 23 & 64 & 1,66 \\
\hline 3 & Sujeto C & 19 & 62 & 1,60 \\
\hline & Promedio & $\mathbf{2 0}$ & $\mathbf{6 2}$ & $\mathbf{1 , 6 2}$ \\
\hline
\end{tabular}

Tabla 2. Variables básicas antropométricas de los bailarines hombres del grupo de danza de la Universidad del Atlántico.

\begin{tabular}{|c|c|c|c|c|}
\hline \multicolumn{5}{|c|}{ SEXO MASCULINO } \\
\hline $\mathbf{N}^{\circ}$ & SUJETOS & EDAD & PESO (kg) & TALLA(m) \\
\hline 1 & Sujeto D & 24 & 63,5 & 1,75 \\
\hline 2 & Sujeto E & 29 & 73,7 & 1,75 \\
\hline 3 & Sujeto F & 24 & 57 & 1,68 \\
\hline 4 & Sujeto G & 27 & 72 & 1,74 \\
\hline & Promedio & $\mathbf{2 6}$ & $\mathbf{6 7}$ & $\mathbf{1 . 7 2}$ \\
\hline
\end{tabular}

\section{Resultados de la relación Cintura / Cadera (Ci/Ca). EI} perímetro de la cintura de las tres mujeres bailarinas oscila entre 73 y $78 \mathrm{~cm}$, con un promedio de $75 \mathrm{~cm}$. Por su parte, el perímetro de la cadera, oscila entre 98 a $105 \mathrm{~cm}$, con un promedio de $101,67 \mathrm{~cm}$. Se encontró que la circunferencia de la cadera de las bailarinas oscila entre 98 y $105 \mathrm{~cm}$, con un promedio de 101,67. La circunferencia del abdomen oscila entre 85 y $92 \mathrm{~cm}$, con un promedio de 87,67 . Por lo tanto, el porcentaje de grasa (\% Gc) oscila entre 31 y 35 , con un promedio de 33,07, una desviación estándar de 2,32 y un coeficiente de variación de $7 \%$.

Teniendo en cuenta, los datos anteriores, se encuentra la relación cintura/cadera $(\mathrm{Ci} / \mathrm{Ca})$, que está entre 0,695 y 0,765, con un promedio de 0,738, una deviación estándar de 0,04 y un coeficiente de variación bajo, de $5 \%$. Según estos datos y teniendo en cuenta, la clasificación de la norma porcentual, ninguna de las bailarinas se encuentra en zona de riesgo, es decir, están en una clasificación normal.

Por otro lado, en los cuatro hombres, el peso oscila entre 63,5 y 73,7 kilos, con un promedio de 67 kilos. La circunferencia del abdomen oscila entre 75 y $90 \mathrm{~cm}$, con un promedio de $79 \mathrm{~cm}$. La circunferencia de la muñeca oscila entre 17 y $18 \mathrm{~cm}$, con un promedio de 18 .
Resultados del índice de estatura al cuadrado (MCM). El peso corporal de las bailarinas oscila entre 60 y $64 \mathrm{~kg}$, con un promedio para las mujeres de $62 \mathrm{~kg}$. La talla, se encuentra entre 63 y 65 pulgadas, con un promedio de 63,91; teniendo en cuenta los datos anteriores, se halla un Índice de Estatura al cuadrado (MCM) entre 44,09 y 49,06, con un promedio de 46,60, una desviación estándar de 2,26 y un coeficiente de variación bajo de $5 \%$. Según estos datos, se halla un porcentaje de grasa que oscila entre 23,18 y 28,00 , una desviación estándar de: 2,74 y un coeficiente de variación medio de $11 \%$.

En cuanto a los cuatro hombres, se halló que el peso corporal oscila entre 57 y $73,7 \mathrm{~kg}$, con un promedio de $67 \mathrm{~kg}$. La talla, se encuentra entre 66 y 69 pulgadas, con un promedio de 68; por eso, se determina un Índice de Estatura al cuadrado (MCM), entre 49,58 y 62,47, con un promedio de 57. Según estos datos, se halla un porcentaje de grasa que oscila entre 13,01 y 16,01. En ellos, la desviación estándar oscila entre 1 y 8 y un coeficiente de variación bajo y medio, entre 2 y $12 \%$.

Resultados de los pliegues cutáneos en mujeres. Para las mujeres, se tomaron unas muestras sobre 3 pliegues cutáneos, que fueron: tricipital, con los 
datos que oscilan entre 13 y $21 \mathrm{~mm}$, con un promedio de $16,67 \mathrm{~mm}$; suprailíaco, los datos oscilan entre 17 y $25 \mathrm{~mm}$, con un promedio de $20,67 \mathrm{~mm}$ y muslo anterior, con los datos que oscilan entre 20 y $32 \mathrm{~mm}$, con un promedio de 27,33 .

La sumatoria de estos tres pliegues permite revelar unos datos, que oscilan entre 53 y 78 , con un promedio de 64,67, una desviación estándar de 12,58 y un coeficiente de variación media de $19 \%$. En síntesis, se observa que, en la desviación estándar, tanto tricipital como suprailíaco, mantienen los valores iguales de 4,04, a diferencia del muslo anterior, con un valor de 6,43. Según esto, teniendo en cuenta, que el coeficiente de variación es la diferencia entre la desviación estándar y el promedio, se observa que la variación es grande en los 3 pliegues cutáneos, tricipital, con $24 \%$; el suprailíaco, con $20 \%$ y el muslo anterior, con $24 \%$.

Las muestras de los pliegues cutáneos de las mujeres oscilan con las siguientes cifras: pecho, entre $8 \mathrm{y}$ $11 \mathrm{~mm}$, con un promedio de 9,67; axilar, entre $15 \mathrm{y}$ $21 \mathrm{~mm}$, con un promedio de 18,67; tricipital, entre 13 y $21 \mathrm{~mm}$, con un promedio de 16,67; subescapular, entre 15 y $16 \mathrm{~mm}$, con un promedio de 15,33; abdominal, entre 25 y $30 \mathrm{~mm}$, con un promedio de 27,67 ; suprailíaco, entre 17 y $25 \mathrm{~mm}$, con un promedio de 20,67; muslo anterior, entre 20 y $32 \mathrm{~mm}$, con un promedio de 27,33 . La sumatoria de estos siete pliegues devela que los resultados están entre 121 y 155 , con un promedio de 136,00 , una desviación estándar de 17,35 y un coeficiente de variación media de $13 \%$. Por lo tanto, la desviación estándar en los 7 pliegues oscila entre 0,58 y 6,43.
Resultados de los pliegues cutáneos en hombres.

En cuanto a los hombres, se realizó la misma toma de muestra sobre 3 pliegues cutáneos: tricipital, los datos oscilan entre 5 y $10 \mathrm{~mm}$, con un promedio de 6; suprailíaco, los datos oscilan entre 5 y $20,1 \mathrm{~mm}$, con un promedio de 14; muslo anterior, los datos oscilan entre 5,1 y $15 \mathrm{~mm}$, con un promedio de 10 . En éstos, la sumatoria de los tres pliegues revela unos datos, que oscilan entre 15,1 y 45,1 con un promedio de 30 , una desviación estándar de 15 y un coeficiente de variación grande de $48 \%$.

En síntesis, se observa que la desviación estándar en el pliegue cutáneo tricipital es de 2 , suprailíaco es de 6 y en el muslo anterior, con un valor de 8; según ésto y sabiendo que el coeficiente de variación es la diferencia entre la desviación estándar y el promedio, se observa que la variación es grande en los 3 pliegues cutáneos; tricipital, con $39 \%$, el suprailíaco, con $54 \%$ y el muslo anterior, con $56 \%$.

En los cuatro bailarines, los datos oscilan de la siguiente forma: pecho, entre 5 y $10 \mathrm{~mm}$, con un promedio de 6; axilar, entre 5,1 y $10,2 \mathrm{~mm}$, con un promedio de 6 ; tricipital, entre 5 y $10 \mathrm{~mm}$, con un promedio de $8 \mathrm{~mm}$; subescapular, entre 5,3 y $10,2 \mathrm{~mm}$, con un promedio de 9; abdominal, entre $5 \mathrm{y}$ $20,1 \mathrm{~mm}$, con un promedio de 14; suprailíaco, entre 5 y $10,3 \mathrm{~mm}$, con un promedio de $8 \mathrm{~mm}$; muslo anterior, entre 5,1 y $15 \mathrm{~mm}$, con un promedio de $10 \mathrm{~mm}$.

Por lo anterior, la sumatoria de estos siete pliegues muestra un resultado que varía entre 40,2 y 85,8 , con un promedio de 61, una desviación estándar de 23 y un coeficiente de variación grande de $38 \%$ (Tablas 3 y 4 ).

Tabla 3. Resultados de los pliegues cutáneos de las bailarinas.

\begin{tabular}{|c|c|c|c|c|c|c|c|c|c|}
\hline SUJETOS & PECHO & AXILA & TRICEP & $\begin{array}{c}\text { SUB } \\
\text { ESCAPULA }\end{array}$ & ABDOMEN & SUPRAILIACO & $\begin{array}{c}\text { MUSLO } \\
\text { ANTERIOR }\end{array}$ & IMC & $\begin{array}{c}\text { MCM } \\
\text { (kg) }\end{array}$ \\
\hline Sujeto A & 10 & 15 & 16 & 15 & 28 & 17 & 20 & 23,15 & 46,09 \\
\hline Sujeto B & 11 & 20 & 21 & 16 & 30 & 25 & 32 & 23,23 & 49,06 \\
\hline Sujeto C & 8 & 21 & 13 & 15 & 25 & 20 & 30 & 24,22 & 44,64 \\
\hline
\end{tabular}

Tabla 4. Resultados de los pliegues cutáneos de los bailarines.

\begin{tabular}{|c|c|c|c|c|c|c|c|c|c|}
\hline SUJETOS & PECHO & AXILA & TRICEP & $\begin{array}{c}\text { SUB } \\
\text { ESCÁPULA }\end{array}$ & ABDOMEN & SUPRAILIACO & $\begin{array}{c}\text { MUSLO } \\
\text { ANTERIOR }\end{array}$ & IMC & $\begin{array}{c}\text { MCM } \\
(\mathrm{kg})\end{array}$ \\
\hline Sujeto D & 5 & 5,1 & 5 & 10 & 5 & 5 & 5,1 & 20,73 & 53,33 \\
\hline Sujeto $\mathrm{E}$ & 10 & 10,2 & 10 & 10,2 & 20,1 & 10,3 & 15 & 24,07 & 62,47 \\
\hline Sujeto $\mathrm{F}$ & 5,2 & 5,2 & 5,3 & 5,3 & 10,2 & 5,2 & 5,2 & 20,20 & 49,58 \\
\hline Sujeto G & 5 & 5,3 & 10 & 10,2 & 20,1 & 10,3 & 15 & 23,78 & 61,76 \\
\hline
\end{tabular}


Porcentaje Grasa Corporal por la sumatoria de 3 pliegues. Para las bailarinas, se obtuvo un porcentaje de grasa, que oscila entre 21,06 y $29,26 \%$, con un promedio de 24,91, una desviación estándar de 4,12 y un coeficiente de variación medio de $17 \%$. Teniendo en cuenta, que los datos de la desviación estándar oscilan entre 0,01 y 12,58 , se observa que la variación es baja, con un valor de $1 \%$.

En los cuatro hombres, se obtuvo un porcentaje de grasa, que oscila entre 3,68 y $13,53 \%$, con un promedio de 9 , una desviación estándar de 5 y un coeficiente de variación grande de 59\%. En síntesis, se observa que los datos de la desviación estándar oscilan entre 0 y 15 ; la variación es baja en la edad, con $9 \%$ y en $\operatorname{Dc} \Sigma 3$, con $1 \%$, a diferencia de $\Sigma 3$, con una variación grande de $48 \%$

\section{Porcentaje de Grasa Corporal por la sumatoria de 7}

pliegues. Para las tres bailarinas, se halló que $\operatorname{Dc} \sum 7$ oscila entre 1,03 y 1,05, con un promedio de 1,04. Teniendo en cuenta todos los datos anteriores, se obtiene un porcentaje de grasa, que oscila entre 23,21 y $28,40 \%$, con un promedio de 25,50 , una desviación estándar de 2,65 y un coeficiente de variación bajo, de $10 \%$. Se observa que los datos de la desviación estándar oscilan entre 0,01 y 17,35. Asimismo, es posible afirmar que la variación es baja en la $\operatorname{Dc} \sum 7$, con un valor de $1 \%$, a diferencia de la edad y la $\Sigma 7$, con $13 \%$ ambas.

En cuanto a los bailarines del grupo, los datos mostraron un porcentaje de grasa entre 4,76 y $12,46 \%$, con un promedio de 8 , una desviación estándar de 4 y un coeficiente de variación grande de $48 \%$. De esta manera, es posible afirmar, que los datos de la desviación estándar oscilan entre 0 y 23, por lo tanto, la variación es baja en la edad, con $9 \%$ y en $\operatorname{Dc} \Sigma 7$, con $1 \%$, a diferencia de $\Sigma 7$, con una variación grande de $38 \%$

Resultados del test fuerza flexo extensión de codos. Las 3 bailarinas mujeres presentaron unos valores que se hallaron entre 11 y 28 repeticiones realizadas, con un promedio de 20 repeticiones, en estas participantes. Tanto la desviación estándar, con 9, como en el coeficiente de variación grande, con $43 \%$, reflejan que hay una alta dispersión en los datos. En los 4 hombres que se evaluaron, se hallaron valores entre 21 y 36 repeticiones realizadas, con un promedio de 28 repeticiones, en estos participantes.
Tanto la desviación estándar, con 6, como en el coeficiente de variación grande, con $22 \%$, reflejan que hay una alta dispersión en los datos.

Resultados del test fuerza abdominales. Las 3 bailarinas mujeres presentaron los siguientes resultados: se hallaron entre 14 y 23 repeticiones realizadas, con un promedio de 19 repeticiones. Tanto la desviación estándar, con 5 , como en el coeficiente de variación grande, con $24 \%$, reflejan que hay una alta dispersión en los datos. En los 4 bailarines hombres, se identificaron valores entre 21 y 27 repeticiones realizadas, con un promedio de 23 repeticiones. Tanto la desviación estándar, con 3 , como en el coeficiente de variación medio, con 13\%, reflejan que hay una mediana dispersión en los datos.

Resultados del test fuerza salto alto. Las 3 bailarinas mujeres presentaron los siguientes resultados: los datos del salto alto oscilan entre 0,37 y 0,46 , con un promedio de 0,42 , con una desviación estándar de 0,05 y un coeficiente de variación medio, de $11 \%$. Los 4 bailarines hombres presentaron los siguientes resultados del salto alto, que oscilan entre 0,40 y 0,64 , con un promedio de 0,49 , una desviación estándar de 0,11 y un coeficiente de variación medio de $23 \%$.

Resultados del test velocidad skipping. Según Grosser (1992) es la capacidad para efectuar un movimiento o una habilidad motriz en el menor tiempo posible. Este tipo de velocidad tiene un gran componente de coordinación. En esta prueba, los valores de las mujeres se hallaron entre 22 y 34 repeticiones realizadas, con un promedio de 26 , una desviación estándar de 7 y un coeficiente de variación grande, de $27 \%$. Teniendo en cuenta lo anterior y haciendo una valoración del tiempo teórico, a través de una regla de tres, se obtienen unos valores, que oscilan entre 5,88 y 9,09 , con un promedio de 8 , una desviación estándar de 2 y un coeficiente de variación grande de $23 \%$.

En los 4 hombres, los valores que se hallaron están entre 38 y 42 repeticiones realizadas, con un promedio de 40, una desviación estándar de 2 y un coeficiente de variación bajo, de $6 \%$. Teniendo en cuenta lo expuesto y haciendo una valoración del tiempo teórico, a través de una regla de 3 , se obtienen unos valores, que oscilan entre 4,76 y 5,26 , con un promedio de 5 , una desviación estándar de 0 y un coeficiente de variación bajo de $6 \%$. 
Resultados del test resistencia aeróbica Cooper. Los resultados de las mujeres, se ubicaron entre 1.700 y 2.000 metros, con un promedio de 1.833 metros, una desviación estándar de 153 y un coeficiente de variación bajo, de $8 \%$. Según lo anterior y la fórmula estándar, para calcular el $\mathrm{ml} / \mathrm{kg} / \mathrm{min}$, se obtuvo un valor, que oscila entre 26,58 y 33,24 , con un promedio de 30 , una desviación estándar de 3 y un coeficiente de variación medio de $11 \%$. Luego de tener estos datos, se puede hacer una valoración, en la cual, se observa que dos mujeres tuvieron un resultado positivo y solo una de ellas, obtuvo un resultado negativo.

Por su parte, los hombres alcanzaron entre 2.050 y 2.650 metros, con un promedio de 2.250, una desviación estándar de 274 y un coeficiente de variación medio, de $12 \%$. Por lo tanto, los resultados generales están entre 34,36 y 47,69 , con un promedio de 39, una desviación estándar de 6 y un coeficiente de variación medio de 16\%. Luego de tener estos datos, se puede hacer una valoración, en la cual, se observa que un sujeto obtuvo un índice muy bueno; dos, tuvieron un resultado de regular y el último, un resultado bueno.

Resultados del test de flexibilidad Wells. Los resultados de las 3 bailarinas mostraron indicadores entre +13 y +16 centímetros, con un promedio de 14, una desviación estándar de 2 y un coeficiente de variación medio, de $12 \%$, por lo cual, se considera que todas tuvieron un resultado positivo. En los hombres, los índices se ubicaron entre +10 y +16 centímetros, con un promedio de 13 , una desviación estándar de 3 y un coeficiente de variación medio, de $20 \%$. Es posible afirmar que, dichas cifras, también fueron positivas.

\section{DISCUSIÓN}

Se contrastaron los resultados hallados en esta investigación con resultados de otros estudios, encontrándose los siguientes referentes. Arana et al. (2013) establecen en su estudio que la edad de la población fluctuó entre 16 y 26 años, para los hombres, con una media de 21 y en mujeres, la edad osciló entre 15 y 22 años, con una media de 18, lo que presenta una gran similitud con los resultados de los bailarines de la Universidad del Atlántico que, en mujeres, fluctúo entre 18 y 23 años, con un promedio de 20 años y los hombres oscilan 24 y 29 años, con un promedio de 26 años, es decir, se trata de población joven.

En otro estudio, Betancourt et al. (2007) muestran los valores de la composición corporal para un peso promedio de 48,90kg; el porcentaje promedio de grasa corporal de las bailarinas fue de $17,73 \%$, equivalente a $8,66 \mathrm{~kg}$ de masa grasa. Por sustracción, la masa corporal activa fue de 40,24kg. En lo que corresponde a los bailarines, con un peso promedio de $67,84 \mathrm{~kg}$, el porcentaje graso fue $8,85 \%$, para un total de $6,00 \mathrm{~kg}$ de masa grasa, mientras que la masa corporal activa fue de $61,84 \mathrm{~kg}$. Se puede observar, que no existe similitud con los datos obtenidos de los bailarines del grupo de danzas de la Universidad del Atlántico, en los que se obtiene un porcentaje de grasa, que oscila entre 23,21 y $28,40 \%$, con un promedio de 25,50; en cuanto a los bailarines del grupo, los datos muestran un porcentaje de grasa entre 4,76 y $12,46 \%$, con un promedio de 8 , una desviación estándar de 4 y un coeficiente de variación grande de $48 \%$.

Según Vargas (2009), comparando diferentes modalidades de danza, se ha observado que los profesionales de danza moderna muestran mejores resultados de fuerza que los de ballet clásico, pero ambos muestran peores resultados, que deportistas de la misma edad. El hecho que bailarines obtengan menores niveles de fuerza que deportistas, no solo es debido a que no realizan un entrenamiento sistematizado de la fuerza, sino que se ve acrecentado, porque las pruebas de valoración de la fuerza son más acordes a patrones motrices familiares para deportistas y no son específicas, para las distintas modalidades de danza; esto está acorde con los resultados de los test de fuerza aplicados a los bailarines de la Universidad del Atlántico, en los cuales, los resultados no fueron los mejores, en los que se observa bastante deficiencia en la capacidad de fuerza.

Enla preparación delos bailarineses necesariotrabajar todas las capacidades físicas durante su práctica diaria, pero hay que tener en cuenta, que todos los organismos son distintos y tienen necesidades específicas, por lo tanto, el entrenamiento físico, se debe enfocar al acondicionamiento aeróbico, sin descuidar las demás capacidades físicas, como la fuerza, la velocidad y la flexibilidad. Debido a las extensas jornadas de ensayos y de presentaciones, la 
fatiga es un factor que puede afectar más rápidamente y contribuir a las lesiones, por lo cual, se recomienda descanso suficiente entre entrenamientos. Tal como mencionan Koutedakis \& Jamurtas (2004), existe una mejora de las cualidades físicas básicas, tras periodos de descanso.

\section{CONCLUSIONES}

En la variable del IMC, se encontró que los bailarines, mujeres y hombres, presentan valores normales, es decir, que todos tenían valores entre 20,20 y 24,22 , en este indicador. Por lo tanto, los resultados están acordes, con lo que se espera de este tipo de población, debido a que la danza exige que los bailarines tengan una composición corporal magra.

Se halló un \% Gc $\sum 3$ para las danzantes, de 24,91\% y para los bailarines, de $9 \%$; un $\% \mathrm{Gc} \Sigma 7$, para mujeres, de $25,50 \%$ y para hombres, de $8 \%$. Si bien los bailarines obtienen una clasificación de peso normal en su índice de masa corporal, el porcentaje de grasa es elevado en mujeres, debido a que existe una diferencia, según la $\Sigma 7$, de $17,5 \%$.

En cuanto a los resultados promedios del test de fuerza, cabe destacar que se realizaron 3 pruebas diferentes: flexo extensión de codos para mujeres, con 20 repeticiones y para hombres, 28 repeticiones; abdominales para mujeres, 19 repeticiones y para hombres, 23 repeticiones; salto alto para mujeres, $0,42 \mathrm{~m}$ y para hombres, $0,49 \mathrm{~m}$.

En el test de resistencia, los resultados fueron: para mujeres, de $30 \mathrm{ml} / \mathrm{kg} / \mathrm{min}$ y para hombres, de $39 \mathrm{ml} /$ $\mathrm{kg} / \mathrm{min}$. Finalmente, en el test de flexibilidad los datos promedios de las mujeres fueron de $+14 \mathrm{~cm}$, mientras que para los hombres fueron de $+13 \mathrm{~cm}$. Por lo anterior, independientemente del nivel de entrenamiento, el talento, la forma de la danza, el sexo o la edad, se considera que los bailarines deberían prevenir el entrenamiento excesivo, con el ánimo de conservar su energía y de evitar el agotamiento.

Se recomienda implementar una dieta saludable, con la cual, se pueda reducir el porcentaje de grasa corporal, por lo tanto, se sugiere acudir a la consultoría que brinda el programa de nutrición de la institución. Finalmente, es indispensable realizar un monitoreo continuo a la condición corporal de los bailarines.
Conflictos de intereses: El manuscrito fue preparado y revisado con la participación de todos los autores, quienes declaramos que no existe ningún conflicto de intereses que ponga en riesgo la validez de los resultados presentados. Financiación: Este estudio fue financiado con recursos propios de los autores del trabajo.

\section{REFERENCIAS}

1. ARANA, T.; VELÁSQUEZ, J.; CARVAJAL, R. 2013. Determinación de la capacidad y la carga física de trabajo en bailarines de una escuela de baile de la ciudad de Cali. Ciencia \& Salud. 1(4):11-16.

2. ARELLANO, D.; YÁÑEZ, J. 2009. Mediciones Antropométricas sin contactos a partir de fotografías. Ide@sConcyteg. 4(48):669-673.

3. BETANCOURT, H.; ALBIZU, J.; DÍAZ, M. 2007. Composición corporal de bailarines élites de la compañía ballet nacional de cuba. Revista Cubana Aliment Nutr. 17(1):8-22

4. BETANCURT, L.; DÍAZ, M. 2006. Estudio longitudinal de la composición Corporal de bailarines cubanos de ballet. Rev. Argentina de Antropología. 8(1):23-35.

5. BOUGART, M. 1966. Técnica de la danza. 5ta ed. Editorial Universal (Buenos Aires, Argentina).

6. BRONNER, S.; OJOFEITIMI, S.; SPRIGGS, J. 2003. Occupational musculoskeletal disorder in dancers. Physical Therapy Reviews. 8(2):57-68. https://doi.org/10.1179/108331903225002416

7. CABALleRO, T. 2015. La Danza y su Demanda Física. Danzando, que es Gerundio. Disponible desde Internet en:

http://expresioncorporalydanzab.blogspot.com. co/2015/04/la-danza-y-su-demanda-fisica.html

8.GARDOQUI, M.;SIERRA, M. 1994. Conceptualización y tratamiento de la expresión corporal como materia de la especialidad de E.F. en las Facultades de Educación, formación del profesorado. Actas I Congreso Nacional de Educación Física de Facultades de Ciencias de la Educación y XII de E.U. de Magisterio. Wanceulem (Sevilla, España).

9. GEORGE, D.; GARTH, A.; VEHRS, P. 2007. Test y Pruebas Físicas. $4^{\circ}$ Edición. Editorial Paidotribo (Barcelona, España). 310p. 
10. GROSSER, M.; SIMON, W.; LLEDÓ, I. 1992. Entrenamiento de la velocidad : fundamentos, métodos y programas. Martínez Roca. Barcelona, España. 220p.

11. HERNÁNDEZ, R.; FERNÁNDEZ, C.; BAPTISTA, P. 2007. Metodología de la investigación. Cuarta edición. McGraw Hill (México). 839p.

12. IRVINE, S.; REDDING, E.; RAFFERTY, S. 2011. La condición física en la danza. International Association for Dance Medicine and Science. Disponible desde Internet en: www.DanceScience.org

13. KOUTEDAKIS, Y.; JAMURTAS, A. 2004. The dancer as a Performin Athlete: Physiological Considerations. SportsMed. 34(10):651-661. https://doi.org/10.2165/00007256-20043410000003

14. LORENZO, F. 2015. Test de Fuerza muscular. Fitness guía. Disponible desde Internet en: http://www.fitnessguia.com/test/fuerzaresistencia/test-fuerza-muscular.html

15. MALINA, R.; BOUCHARD, C. 1991. Growth, maturation, and physical activity. Human Kinetic Pub. 712p.

16. MARCHENA, V.; SOLÓRZANO, P., DUEÑAS, M. 2008. La preparación física en estudiantes de danza de las escuelas de artes. Efdeportes. 13(122)

17. MAZZA, J.C. 2000. Antrometrica. Byosystem (Argentina). Edición original: Norton, K.; Olds, T. Anthropometrica. University of New South Wales Press (Australia). 274p.

18. PATE, R.; ORIA, M.; PILLSBURY, L. 2012. Fitness Measures for a National Youth Survey. National Academies Press (Washington). 274p.

19. POLLOCK, M.L.; WILMORE, J.H.; FOXIII, S.M. 1990. Exercise in health and disease: Evaluation and prescription for prevention and rehabilitation. 2 edition. Saunder Company. 741p.

20. ROBERGS, R.; ROBERTS, S. 1999. Fundamental principles of exercise physiology for fitness, performance, and health. McGraw-Hill (Boston). 487p.

21. ROSS, W.; KERR, D. 1991. Fraccionamiento de la masa corporal: un nuevo método para utilizar en nutrición clínica y medicina deportiva. Apunts: Educación física y deportes. 18:175-187.
22. SÁNCHEZ, M. 2016. La preparación Física de un Bailarín. ABC Danzar. Disponible desde Internet en:

https://abcdanzar.blogspot.com.co/2016/02/ la-preparacion-fisica-de-un-bailarin.html

23. SIRI, W.E. 1961. Body composition from fluid spaces and density: analysis of methods. En: Brozeck, J.; Henschel, A. eds. Techniques for measuring body composition. Washington DC: National Academy of Sciences. Natural Resourcer Council. 33p.

24. WELLS, K.F.; DILLON, E.K. 1952. The Sit and Reach - A Test of Back and Leg Flexibility, Research Quarterly. American Association for Health, Physical Education and Recreation. 23(1):115-118. https://doi.org/10.1080/10671188.1952.10 761965

25. VARGAS, A. 2009. Danza y condición física. Revista del Centro de Investigación FlamencoTelethusa. 2(2):16-24.

26. VICENTE, M.A. 2015. Desarrollo de un sistema de captura de siluetas en Android. Proyecto Final de Carrera, Universidad Politécnica de Valencia.

27. YASUMURA, S.; WANG, J.; PIERSON, R. 2000. In vivo composition studies (Annals of the New York Academy of Sciences). New York Academy of Sciences (New York, NY). 637p. 